\section{J.A. Caminero}

Pneumology Dept, General Hospital of Gran Canaria “Dr. Negrín", Barranco de la Ballena s/n, Las Palmas, Spain.

\section{STATEMENT OF INTEREST}

None declared.

\section{REFERENCES}

1 Migliori GB, Besozzi G, Girardi E, et al. Clinical and operational value of the extensively drug-resistant tuberculosis definition. Eur Respir J 2007; 30: 623-626.

2 Migliori GB, Lange C, Centis R, et al. Resistance to secondline injectables and treatment outcomes in multidrugresistant and extensively drug-resistant tuberculosis cases. Eur Respir J 2008; 31: 1155-1159.

3 World Health Organization. The global MDR-TB \& XDRTB response plan 2007-2008. Geneva, World Health Organization, 2007.

4 Raviglione MC, Smith IM. XDR tuberculosis - implications for global public health. N Engl J Med 2007; 356: 656-659.

5 Gandhi NR, Moll A, Sturm AW, et al. Extensively drugresistant tuberculosis as a cause of death in patients coinfected with tuberculosis in a rural area of South Africa. Lancet 2006; 368: 1575-1580.

6 Centers for Disease Control and Prevention. Emergence of Mycobacterium tuberculosis with extensive resistance to second-line drugs - worldwide, 2000-2004. Morb Mortal Wkly Rep 2006; 55: 301-305.

7 Jeon CY. Extensively drug-resistant tuberculosis in South Korea: risk factors and treatment outcome among patients at a tertiary referral hospital. Clin Infect Dis 2008; 46: 42-49.
8 Tousek J, Jancik E, Zelenka M, Jancikova-Máková M. The results of treatment in patients with cultures resistant to streptomycin, isoniazid and PAS, a five-year follow-up. Tubercle 1967; 48: 27-31.

9 Zierski M, Zachara A. Late results in re-treatment of patients with pulmonary tuberculosis. Tubercle 1970; 51: 172-177.

10 Somner AR, Brace AA. Late results of treatment of chronic drug-resistant pulmonary tuberculosis. BMJ 1966; 1: 775-778.

11 Fischer DA, Lester W, Dye WE, Moulding TS. Re-treatment of patients with isoniazid-resistant tuberculosis. Analysis and follow-up of 146 cases. Am Rev Respir Dis 1968; 97: 392-398.

12 Kass I. Chemotherapy regimens used in retreatment ofpulmonary tuberculosis. I. Observations on the efficacy of combinations of kanamycin, ethionamide and either cycloserine or pyrazinamide. Tubercle 1965; 46: 151-165.

13 Pines A. Treatment of pulmonary tuberculosis with cultures resistant to two or more drugs: a series of 44 patients. Tubercle 1965; 46: 131-142.

14 Kass I. Chemotherapy regiments used in retreatment of pulmonary tuberculosis. II. Observations on the efficacy of combinations of ethambutol, capreomycin and companion drugs, including 4-4 diisoamyloxythiosemicarbanilide. Tubercle 1965; 46: 166-177.

15 Caminero JA. Treatment of multidrug-resistant tuberculosis: evidence and controversies. Int J Tuberc Lung Dis 2006; 10: 829-837.

\title{
An ecological analysis of incidence of tuberculosis and per capita gross domestic product
}

\section{To the Editor:}

In 2006, an estimated 9 million new cases of tuberculosis (TB) emerged worldwide [1]. Of these, only $1 \%$ occurred in the European Union and North America combined, while Africa and South-East Asia contributed $>65 \%$. Among the 22 highburden countries, 17 were in Africa, 16 of which were in the lowest quartile in terms of per capita gross domestic product (GDP). Since 2001, the fight against poverty has been a major theme in the World Health Organization's (WHO) "Stop TB" strategy. Indeed, poverty "fuels" TB by facilitating transmission through crowded working and living conditions, it may increase the risk of progression to disease through malnutrition, and imposes barriers to accessing health services.

The aim of the present analysis was to explore and illustrate the relationship between the incidence of TB and an indicator of standard of living (per capita GDP) using WHO and World Bank estimates.
The per capita GDP, i.e. total market value of all final goods and services produced within a given country in a given period of time, is often used as an indicator of standard of living. The advantages are that it is measured frequently, widely used and easily accessible information, and that technical definitions for estimating GDP are relatively consistent between countries.

The World Bank publishes yearly estimates of per capita GDP per country [2]. Similarly, the WHO provides annual estimates for TB incidence for each country by WHO region [2]. Of the 211 WHO member states and 177 countries with reports by the World Bank, there were 171 pairs with information on both GDP and TB. For uniformity of the denominator, United Nations population estimates for 2004 [3] were used to obtain per capita figures for GDP and TB, respectively.

The choice of the same denominator permitted a direct regression on the numerators (rather than on rates), using population size as a weight. Linear regression on the logarithm 


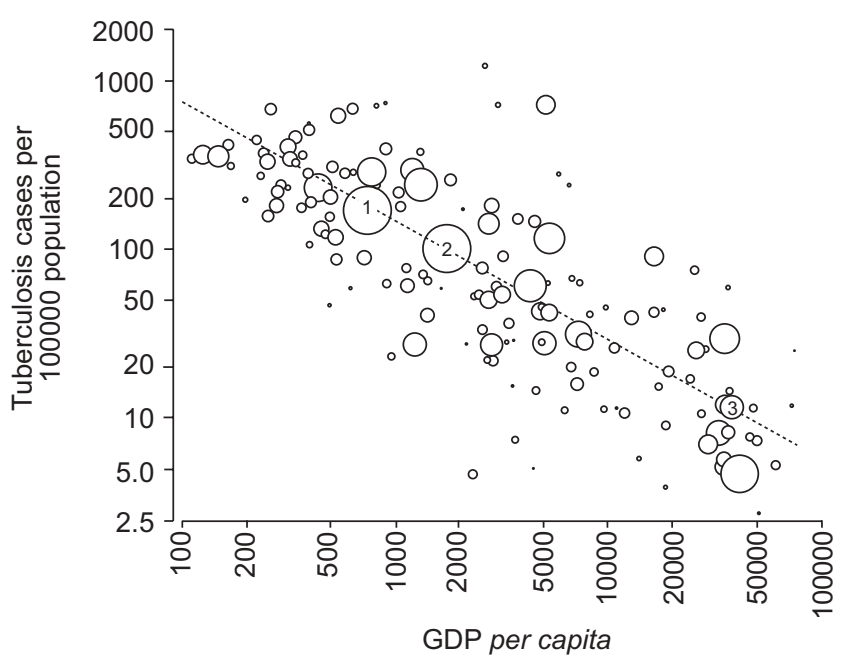

FIGURE 1. Relationship between per capita gross domestic product (GDP; Wold Bank data, 2005) and incidence of tuberculosis per 100,000 population. For graphical presentation, the third root of the population (millions) divided by 10 was used to determine the size of the symbols. This arbitrary choice provides a visual appreciation that large sized populations do not bear an excessive weight at the extremes of the axes, but are rather distributed across the entire scale. Both abscissa and ordinate were drawn logarithmically. 1; India; 2: China; 3: UK.

of both TB (2004) and GDP (2005) was chosen as the intuitively simplest model. Both unweighted (not shown) and weighted regression gave similar results (fig. 1).

To visually convey the lack of influence of population size in figure 1, the size of the circles representing individual countries was varied according to an arbitrarily chosen factor, as directly proportional graphical representation would have resulted in gross visual distortion. Examples for the correlation between GDP and TB are shown in figure 1 for India, China and the UK. The regression analysis suggests that each doubling of GDP was associated with a $38.5 \%$ decrease in the incidence of TB. For instance, the ratio of per capita GDP between India and China is $\sim 2.3$ and the incidence of TB is $40 \%$ lower in China than in India.

As the unit of measurement is a country, this ecological analysis does not allow a conclusion to be reached for individuals. Furthermore, the analysis was not adjusted for HIV infection, largely because estimates of HIV infection are incomplete for many countries. Nevertheless, the take-home message is clear and emphasises a major thrust of the World Health Organization's strategy: to stop tuberculosis, we must fight poverty.

\section{J-P. Janssens* and H.L. Rieder ${ }^{\#}$}

*Division of Pulmonary Diseases, Geneva University Hospitals, Geneva, and "International Union Against Tuberculosis and Lung Disease, Tuberculosis Dept, Kirchlindach, Switzerland.

\section{STATEMENT OF INTEREST}

None declared.

\section{REFERENCES}

1 World Health Organization. Global tuberculosis control: surveillance, planning, financing. WHO report 2006. Geneva, World Health Organization, 2006.

2 The World Bank. http://web.worldbank.org/eternal/ default $/$ main?menuPK=64133165\&pagePK=64133485\&piPK $=64133503 \& g=$ gdp \&theSitePK $=239419$ Date last accessed: September 8, 2008. Date last updated: August 2008.

3 World Population Prospects. The 2004 Revision. Volume III: Analytical Report. New York, United Nations Population Divisions, 2005.

\section{Diagnosis of pulmonary thromboembolism with endobronchial ultrasound}

\section{To the Editors:}

Endobronchial ultrasound (EBUS) is a new addition to the diagnostic armamentaria of the pneumologist. Its properties allow for excellent visualisation of structures surrounding the airways and, as such, have significant potential to add to diagnostic bronchoscopies. EBUS has a definitive role in detection and biopsy of mediastinal lymph nodes or masses
$[1,2]$. However, we believe that the potential use of this procedure is still underestimated. For this reason, in this letter we report on the case of a 26-yr-old male admitted to the Interventional Pneumology Dept of G.B. Morgagni Hospital (Forlí, Italy) because of fever and exertional dyspnoea.

The patient presented with fatigue, blood-tinged sputum and hypoxia (arterial oxygen tension $8.2 \mathrm{kPa}(62 \mathrm{mmHg})$ ). The 